\title{
Elastic Constants and Pressure-Induced Effects in $\mathrm{MoS}_{2}$
}

\author{
H. Peelaers* and C. G. Van de Walle \\ Materials Department, University of California, Santa Barbara, California 93106-5050, United States
}

ABSTRACT: We use hybrid density functional theory, including van der Waals interactions, to study the elastic properties of bulk molybdenum disulfide $\left(\mathrm{MoS}_{2}\right)$. We determine a complete, consistent set of accurate values for elastic constants, overcoming the inconsistencies in the reported values in the literature. We also elucidate the changes in materials properties that occur when hydrostatic pressure is applied. The compression ratio, the transition from semiconductor to semimetal, and the pressure dependence of the elastic constants are discussed. The good agreement with experiments for small hydrostatic pressures validates our methodology and our calculated values for the elastic constants.

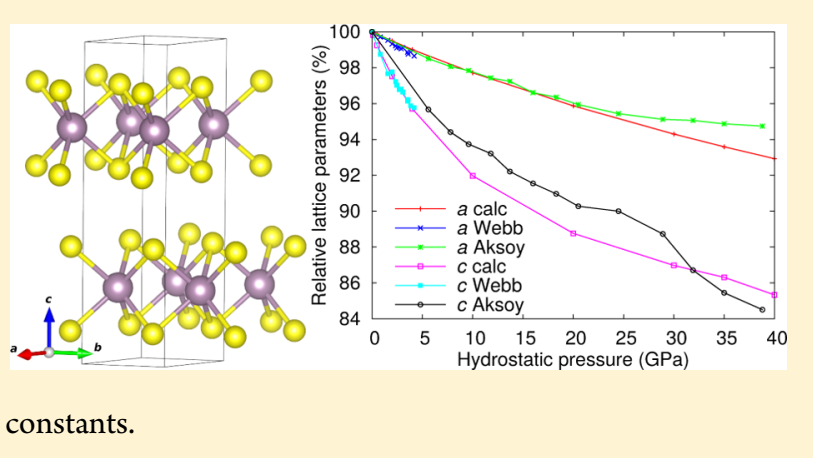

\section{INTRODUCTION}

$\mathrm{MoS}_{2}$ is a layered material in which the layers are weakly bound by van der Waals (vdW) interactions. Recently, it has attracted a lot of attention for use in electronic devices. Monolayers, multilayers, and bulk have all been used in prototype FETs. ${ }^{1-4}$ In this paper we will focus on bulk $\mathrm{MoS}_{2}$. Accurate knowledge of the mechanical properties of $\mathrm{MoS}_{2}$ is important for future device applications, for example, when $\mathrm{MoS}_{2}$ is deposited on a flexible substrate. ${ }^{5}$ Strain significantly affects the electronic structure, and a number of experimental and theoretical studies have already been performed to investigate the effect of strain on the properties of monolayer, bilayer, and bulk $\mathrm{MoS}_{2}{ }^{6-14}$ None of these investigations, however, focused on the elastic properties.

Traditionally, bulk $\mathrm{MoS}_{2}$ is used as a dry lubricant, because of its very low friction coefficients. ${ }^{15}$ These lubricating properties are retained under high pressures. The elastic constants determine the utility of a material as a lubricant. ${ }^{16}$ Elastic constants enter into the relationship linking strain (deformation) of a material and the stress/pressure applied to it. In a hexagonal material such as $\mathrm{MoS}_{2}$ there are five independent elastic constants. An experimental determination was reported by Feldman ${ }^{17}$ based on combining neutron data ${ }^{18}$ and linear compressibilities. ${ }^{19}$ However, there are substantial differences between the resulting values and theoretical calculations. ${ }^{20-22}$ The reported theoretical values also display significant inconsistencies.

Here we address this issue by performing state-of-the-art hybrid functional calculations of the elastic constants in bulk $\mathrm{MoS}_{2}$. The vdW interactions are explicitly included. We also specifically investigate the effects of hydrostatic pressure, shedding light on experiments. Consequences for the electronic structure are also discussed.

\section{COMPUTATIONAL METHODS}

van der Waals interactions play an important role in $\mathrm{MoS}_{2}$. Traditional functionals in density functional theory (DFT) do not include these interactions. We explicitly include vdW interactions by using the semiempirical Grimme D2 correction, ${ }^{23}$ which describes the interactions by a pairwise force field. To obtain accurate electronic band structures as well as accurate atomic structures, we use the screened hybrid functional of Heyd, Scuseria, and Ernzerhof (HSE06), ${ }^{24,25}$ which overcomes the band gap underestimation inherent in traditional functionals such as the local-density approximation or generalized-gradient approximation (GGA). We will call this combination of HSE06 with the D2 correction HSE06-D2. We also tested the more recent $\mathrm{D} 3{ }^{26}$ method in combination with HSE06. The results obtained with D3 are very similar to those obtained with D2, with marginally better performance for the D2 results that are reported here. We use projector augmentedwave pseudopotentials ${ }^{27}$ as implemented in the VASP code, ${ }^{28}$ with a plane-wave cutoff of $400 \mathrm{eV}$ and a $6 \times 6 \times 2$ Monkhorst-Pack k-point grid. ${ }^{29}$ The elastic constants are determined using the stress-strain relations (Hooke's law) by deforming the unit cell. Three different deformation patterns are sufficient to obtain all elastic constants. We use the Voigt notation for the indices.

\section{RESULTS AND DISCUSSION}

The calculated elastic constants are reported in Table 1, where they are compared with experimental ${ }^{17}$ and previously calculated values. ${ }^{20-22}$ None of the other calculations included $\mathrm{vdW}$ interactions. We obtain overall good agreement with experiment, especially for $c_{11}, c_{33}$, and $c_{44}$, which are all determined from neutron scattering data. ${ }^{17}$ For $c_{12}$ we find the opposite sign compared to experiment. Reference 20 reported the same positive sign as we found, while refs 21 and 22 obtained a negative sign; we note that these last two references are from the same group of authors. For $c_{13}$ the range of values

Received: April 15, 2014

Revised: $\quad$ May 9, 2014

Published: May 14, 2014 
Table 1. Elastic Constants (GPa) of $\mathrm{MoS}_{2}$ Obtained Using Different Methods

\begin{tabular}{|c|c|c|c|c|c|c|c|}
\hline & $\begin{array}{l}\text { this work } \\
\text { HSE06-D2 }\end{array}$ & $\exp ^{a}$ & $\mathrm{GGA}^{b}$ & $\mathrm{GGA}^{c}$ & $\mathrm{HF}^{d}$ & $\underset{\mathrm{HF}^{c}}{\text { periodic }}$ & $\mathrm{LDA}^{c}$ \\
\hline$c_{11}$ & 238 & 238 & 211 & 211 & 255 & 218 & 240 \\
\hline$c_{12}$ & 64 & -54 & 49 & -62 & -38 & -21 & -63 \\
\hline$c_{13}$ & 12 & 23 & 3 & 26 & 17 & 39 & 32 \\
\hline$c_{33}$ & 57 & 52 & 37 & 42 & 35 & 59 & 53 \\
\hline$c_{44}$ & 18 & 19 & 30 & 19 & 15 & 30 & 26 \\
\hline
\end{tabular}

${ }^{a}$ Reference $17 .{ }^{b}$ Reference 20. ${ }^{c}$ Reference $21 .{ }^{d}$ Reference 22.

reported by the different theoretical calculations is large, which can be attributed to the lack of inclusion of vdW interactions. Our value differs from experiment by a factor of 2 , though it should be noted that the absolute value of $c_{13}$ is quite small. $c_{12}$ and $c_{13}$ are not determined directly, in experiment, but via the linear compressibilities, and measurement of the latter is probably complicated by the lubricating properties of $\mathrm{MoS}_{2}$. Feldman ${ }^{17}$ used linear compressibility values from ref 19; the latter report an in-plane zero-pressure lattice constant of $3.19 \AA$, substantially larger than that of pure $\mathrm{MoS}_{2}$ (3.16 $\AA$ at room temperature $),{ }^{30}$ creating additional experimental uncertainties.

A number of studies of $\mathrm{MoS}_{2}$ have focused on its behavior under high pressure, because for its application as a lubricant it is important that it maintains its structure under pressure. Diamond anvil cells have been used, in which the samples are subjected to hydrostatic pressure. We compare our results with the experimental results of refs 19 and 31 . Figure 1 shows the

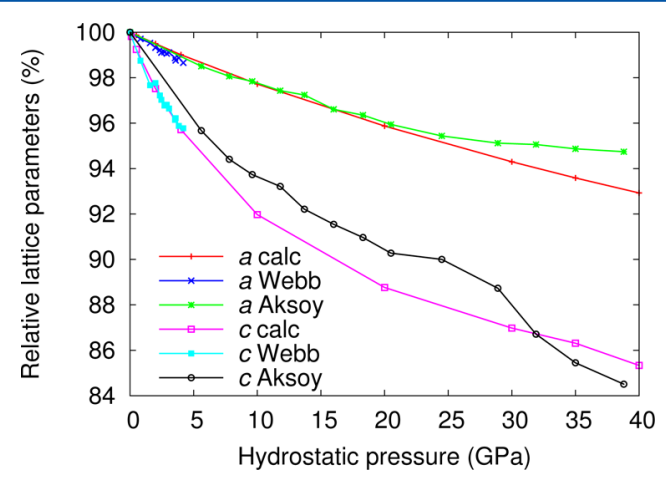

Figure 1. Variation of the lattice constants $a$ and $c$ under hydrostatic pressure. The lattice parameters are referenced to their equilibrium values (corresponding to 100\%). Our results calculated with HSE06D2 are compared with the experiments by Webb et al. ${ }^{19}$ and by Aksoy et $\mathrm{al}^{31}$

lattice parameters expressed as a percentage of the equilibrium lattice parameters for hydrostatic pressures up to $40 \mathrm{GPa}$. This allows comparing $a$ (in-plane) and $c$ (out-of-plane) directly and avoids complications due to the different equilibrium $a$ lattice constant observed by Webb et al. ${ }^{19}$ Our results are in excellent agreement with those of Webb et al., ${ }^{19}$ who reported results up to $4 \mathrm{GPa}$. The agreement with the values of Aksoy et al. ${ }^{31}$ for the $a$ lattice parameter is also very good up to $25 \mathrm{GPa}$; Aksoy et al. stated that a phase transition might occur at this pressure. The $c$ lattice parameter of Aksoy et al. ${ }^{31}$ is larger than the calculated values. The discrepancy may be due to the already mentioned experimental uncertainties, as well as some inconsistencies in ref $31 .^{a}$ Overall, the good agreement, especially at small pressures, validates our calculations and our values for the elastic constants.
More information can be extracted if we plot the ratio of the change in $c$ lattice constant versus the change in a lattice constant. This is equivalent to the ratio of the strains in the $z$ direction versus the $x$ direction. This ratio $\varepsilon_{z z} / \varepsilon_{x x}$, plotted in Figure 2 , is significantly larger than 1 , indicating that the

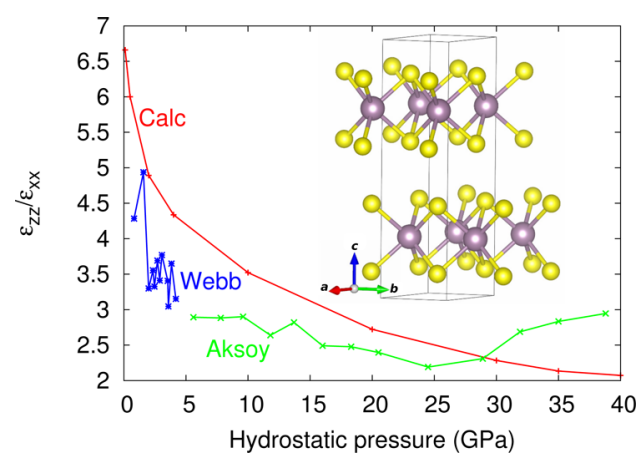

Figure 2. Ratio $\varepsilon_{z z} / \varepsilon_{x x}$ as extracted from our calculations and from the experimental data by Webb et al. ${ }^{19}$ and by Aksoy et al. ${ }^{31}$ The inset shows a ball-and-stick model of $\mathrm{MoS}_{2}$, with the unit cell and axes indicated.

material can be much more easily compressed along the $c$ direction than along the in-plane directions. Both the calculated and experimental results decline monotonically (except for the Aksoy ${ }^{31}$ results above $25 \mathrm{GPa}$, as discussed above). The weak van der Waals interactions between the layers are responsible for this behavior: although initially compression along $c$ is easy, it becomes increasingly more difficult due to an increase in interaction strength between the layers as the interlayer distance increases.

The change in $\varepsilon_{z z} / \varepsilon_{x x}$ observed in Figure 2 is indicative of nonlinear behavior, which means that the elastic constants derived above are only valid in a narrow range of stresses/ pressures near the equilibrium volume. The stress/strain relationships become nonlinear at larger stresses. A full description of this behavior is very complex and beyond the scope of the present investigation; here we focus on the values of elastic constants that correspond to different hydrostatic pressures, which has been the common means of experimental investigation. Figure 3 shows that the calculated elastic constants vary linearly with pressure over the range of hydrostatic pressures up to $40 \mathrm{GPa}$. The slope of the linear fits as a function of the hydrostatic pressure differs substantially between the elastic constants: $c_{11}$ and $c_{12}$ increase by a relatively

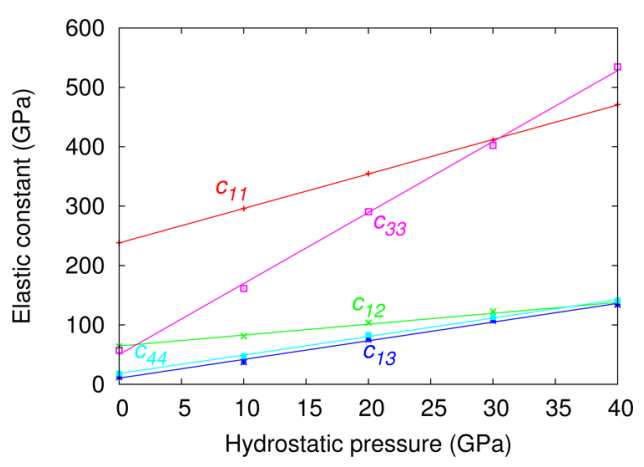

Figure 3. Elastic constants of $\mathrm{MoS}_{2}$ calculated at different hydrostatic pressures. Symbols indicate the calculated values, and the lines are linear fits. 
modest factor of 2 over the pressure range, whereas the other elastic constants increase by almost an order of magnitude.

The application of pressure results in changes in the electronic structure. A very important quantity is the band gap. In some semiconductors, pressure can lead to a decrease in the band gap, ultimately closing the gap, i.e., resulting in a semimetal. We observe this behavior in $\mathrm{MoS}_{2}$, where the band gap closes at a hydrostatic pressure of $35 \mathrm{GPa}$, as shown in Figure 4. This result differs from the value of $25 \mathrm{GPa}$ calculated

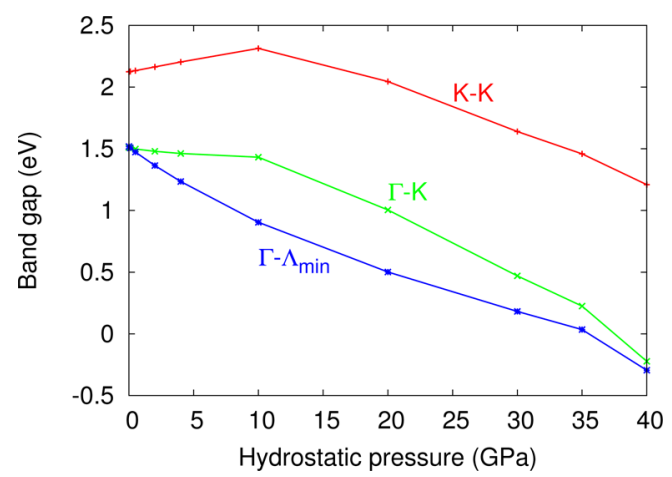

Figure 4. Evolution of the different band gaps of $\mathrm{MoS}_{2}$ as a function of hydrostatic pressure.

by DFT in ref 32; this can be attributed to the use of a GGA functional in that work, which severely underestimates band gaps. It also differs from the work by Wei et al., ${ }^{20}$ who also performed GGA calculations but did not observe a transition to a semimetal at pressures up to $40 \mathrm{GPa}$. The difference between the two GGA results might be explained by the absence of vdW interactions in the work of Wei et al. ${ }^{20}$

The valence-band maximum is located at the $\Gamma$ point over the entire pressure range considered here. At zero pressure, the conduction-band minimum is at the $\mathrm{K}$ point, with the $\Lambda_{\text {min }}$ point (on the high-symmetry $\Gamma-\mathrm{K}$ line) only slightly higher in energy. As soon as pressure is applied, $\Lambda_{\min }$ becomes the lowest energy minimum and remains so over the pressure range up to $40 \mathrm{GPa}$. We can understand this behavior in terms of the two components of pressure: a reduction of the lattice parameters in the in-plane direction leads to a shift of the conduction-band minimum from the $\mathrm{K}$ to the $\Lambda_{\text {min }}$ point. ${ }^{11}$ This is accompanied by a small increase in the magnitude of the band gap, but this is more than compensated by the much larger decrease (Figure 1) in the $c$ lattice parameter, which is known to lead to a decrease in the band gap. ${ }^{11}$ The combination of in-plane and out-ofplane strain thus leads to a decrease in the magnitude of the band gap, with $\Lambda_{\min }$ as the conduction-band minimum. This decrease leads to a transition to a semimetal for a hydrostatic pressure of $35 \mathrm{GPa}$.

\section{CONCLUSIONS}

We have calculated values for the elastic constants of $\mathrm{MoS}_{2}$ based on DFT with a hybrid functional and explicit inclusion of $\mathrm{vdW}$ interactions. We obtain good agreement with experiments for the variation of the lattice parameters under hydrostatic pressure, which provides validation of our methodology. Distinct nonlinearities in the elastic behavior are observed as a function of increasing hydrostatic pressure. For pressures larger than $35 \mathrm{GPa}, \mathrm{MoS}_{2}$ becomes semimetallic.

\section{AUTHOR INFORMATION}

\section{Corresponding Author}

*H. Peelaers: e-mail, peelaers@engineering.ucsb.edu.

Notes

The authors declare no competing financial interest.

\section{ACKNOWLEDGMENTS}

This work was supported by the U.S. Department of Energy, Office of Science, Basic Energy Sciences under Award \#DEFG02-07ER46434. Computational resources were provided by the National Energy Research Scientific Computing Center (NERSC), which is supported by the DOE Office of Science under Contract No. DE-AC02-05CH11231, and by the Extreme Science and Engineering Discovery Environment (XSEDE), supported by NSF (OCI-1053575 and DMR070072N).

\section{ADDITIONAL NOTE}

${ }^{a}$ The values included in Figure 1 are taken from Table 1 of ref 31. These values are different from the ones plotted in Figure 4 of the same reference. The text mentions a consistent decrease in values, in contrast to what is shown in Figure 4. No explanation for theses difference is given in ref 31 .

\section{REFERENCES}

(1) Radisavljevic, B.; Radenovic, A.; Brivio, J.; Giacometti, V.; Kis, A. Single-Layer $\mathrm{MoS}_{2}$ Transistors. Nat. Nanotechnol. 2011, 6, 147-150.

(2) Wang, H.; Yu, L.; Lee, Y.-H.; Shi, Y.; Hsu, A.; Chin, M. L.; Li, L.J.; Dubey, M.; Kong, J.; Palacios, T. Integrated Circuits Based on Bilayer $\mathrm{MoS}_{2}$ Transistors. Nano Lett. 2012, 12, 4674-4680.

(3) Fiori, G.; Szafranek, B. N.; Iannaccone, G.; Neumaier, D. Velocity Saturation in Few- Layer $\mathrm{MoS}_{2}$ Transistor. Appl. Phys. Lett. 2013, 103, 233509.

(4) Kim, S.; Konar, A.; Hwang, W.-S.; Lee, J. H.; Lee, J.; Yang, J.; Jung, C.; Kim, H.; Yoo, J.-B.; Choi, J.-Y.; et al. High-Mobility and LowPower Thin-Film Transistors Based on Multilayer $\mathrm{MoS}_{2}$ Crystals. Nat. Commun. 2012, 3, 1011.

(5) Chang, H.-Y.; Yang, S.; Lee, J.; Tao, L.; Hwang, W.-S.; Jena, D.; $\mathrm{Lu}, \mathrm{N}$.; Akinwande, D. High-Performance, Highly Bendable $\mathrm{MoS}_{2}$ Transistors with High-K Dielectrics for Flexible Low-Power Systems. ACS Nano 2013, 7, 5446-5452.

(6) Scalise, E.; Houssa, M.; Pourtois, G.; Afanas'ev, V.; Stesmans, A. Strain-Induced Semiconductor to Metal Transition in the TwoDimensional Honeycomb Structure of $\mathrm{MoS}_{2}$. Nano Res. 2011, 5, 4348.

(7) Bertolazzi, S.; Brivio, J.; Kis, A. Stretching and Breaking of Ultrathin $\mathrm{MoS}_{2}$. ACS Nano 2011, 5, 9703-9709.

(8) Lu, P.; Wu, X.; Guo, W.; Zeng, X. C. Strain-Dependent Electronic and Magnetic Properties of $\mathrm{MoS}_{2}$ Monolayer, Bilayer, Nanoribbons and Nanotubes. Phys. Chem. Chem. Phys. 2012, 14, 13035-13040.

(9) Yun, W. S.; Han, S. W.; Hong, S. C.; Kim, I. G.; Lee, J. D. Thickness and Strain Effects on Electronic Structures of Transition Metal Dichalcogenides: $2 \mathrm{H}-\mathrm{MoX}_{2}$ Semiconductors $(\mathrm{M}=\mathrm{Mo}, \mathrm{W} ; \mathrm{X}=$ S, Se, Te). Phys. Rev. B 2012, 85, 033305.

(10) Bhattacharyya, S.; Singh, A. K. Semiconductor-Metal Transition in Semiconducting Bilayer Sheets of Transition-Metal Dichalcogenides. Phys. Rev. B 2012, 86, 075454.

(11) Peelaers, H.; Van de Walle, C. G. Effects of Strain on Band Structure and Effective Masses in $\mathrm{MoS}_{2}$. Phys. Rev. B 2012, 86, 241401.

(12) Cheiwchanchamnangij, T.; Lambrecht, W. R. L.; Song, Y.; Dery, H. Strain Effects on the Spin-Orbit-Induced Band Structure Splittings in Monolayer $\mathrm{MoS}_{2}$ and Graphene. Phys. Rev. B 2013, 88, 155404.

(13) Hui, Y. Y.; Liu, X.; Jie, W.; Chan, N. Y.; Hao, J.; Hsu, Y.-T.; Li, L.-J.; Guo, W.; Lau, S. P. Exceptional Tunability of Band Energy in a 
Compressively Strained Trilayer $\mathrm{MoS}_{2}$ Sheet. ACS Nano 2013, 7, $7126-7131$.

(14) Castellanos-Gomez, A.; Roldán, R; Cappelluti, E.; Buscema, M.; Guinea, F.; van der Zant, H. S. J.; Steele, G. A. Local Strain Engineering in Atomically Thin $\mathrm{MoS}_{2}$. Nano Lett. 2013, 13, 53615366.

(15) Martin, J.; Donnet, C.; Le Mogne, T.; Epicier, T. Superlubricity of Molybdenum Disulphide. Phys. Rev. B 1993, 48, 10583-10586.

(16) Gardos, M. In Mechanics of Coatings; Dowson, D., Taylor, C. M., Godet, M., Eds.; Elsevier: Amsterdam, 1990; pp 3-13.

(17) Feldman, J. Elastic Constants of $2 \mathrm{H}-\mathrm{MoS}_{2}$ and $2 \mathrm{H}-\mathrm{NbSe} 2$ Extracted from Measured Dispersion Curves and Linear Compressibilities. J. Phys. Chem. Solids 1976, 37, 1141-1144.

(18) Wakabayashi, N.; Smith, H.; Nicklow, R. Lattice Dynamics of Hexagonal $\mathrm{MoS}_{2}$ Studied by Neutron Scattering. Phys. Rev. B 1975, 12, 659-663.

(19) Webb, A.; Feldman, J.; Skelton, E.; Towle, L.; Liu, C.; Spain, I. High Pressure Investigations of $\mathrm{MoS}_{2}$. J. Phys. Chem. Solids 1976, 37, 329-335.

(20) Wei, L.; Jun-fang, C.; Qinyu, H.; Teng, W. Electronic and Elastic Properties of $\mathrm{MoS}_{2}$. Physica B 2010, 405, 2498-2502.

(21) Todorova, T.; Alexiev, V.; Prins, R.; Weber, T. Ab Initio Study of $2 \mathrm{H}-\mathrm{MoS}_{2}$ Using Hay and Wadt Effective Core Pseudo-Potentials for Modelling the $(10 \overline{1} 0)$ Surface Structure. Phys. Chem. Chem. Phys. 2004, 6, 3023-3030.

(22) Alexiev, V.; Prins, R.; Weber, T. Ab Initio Study of $\mathrm{MoS}_{2}$ and Li Adsorbed on the (1010) Face of $\mathrm{MoS}_{2}$. Phys. Chem. Chem. Phys. 2000, $2,1815-1827$.

(23) Grimme, S. Semiempirical GGA-Type Density Functional Constructed with a Long- Range Dispersion Correction. J. Comput. Chem. 2006, 27, 1787-1799.

(24) Heyd, J.; Scuseria, G. E.; Ernzerhof, M. Hybrid Functionals Based on a Screened Coulomb Potential. J. Chem. Phys. 2003, 118, $8207-8215$.

(25) Heyd, J.; Scuseria, G. E.; Ernzerhof, M. Erratum: "Hybrid Functionals Based on a Screened Coulomb Potential" [J. Chem. Phys. 118, 8207 (2003)]. J. Chem. Phys. 2006, 124, 219906.

(26) Grimme, S.; Antony, J.; Ehrlich, S.; Krieg, H. A Consistent and Accurate Ab Initio Parametrization of Density Functional Dispersion Correction (DFT-D) for the 94 Elements H-Pu. J. Chem. Phys. 2010, 132, 154104.

(27) Blöchl, P. Projector Augmented-Wave Method. Phys. Rev. B 1994, 50, 17953-17979.

(28) Kresse, G.; Furthmüller, J. Efficient Iterative Schemes for Ab Initio Total-Energy Calculations Using a Plane-Wave Basis Set. Phys. Rev. B 1996, 54, 11169-11186.

(29) Monkhorst, H. J.; Pack, J. D. Special Points for Brillouin-Zone Integrations. Phys. Rev. B 1976, 13, 5188-5192.

(30) Murray, R.; Evans, B. The Thermal Expansion of $2 \mathrm{H}-\mathrm{MoS}_{2}$ and 2H-WSe2 between 10 and 320 K. J. Appl. Crystallogr. 1979, 12, 312315.

(31) Aksoy, R; Ma, Y.; Selvi, E.; Chyu, M. C.; Ertas, A.; White, A. XRay Diffraction Study of Molybdenum Disulfide to 38.8 GPa. J. Phys. Chem. Solids 2006, 67, 1914-1917.

(32) Guo, H.; Yang, T.; Tao, P.; Wang, Y.; Zhang, Z. High Pressure Effect on Structure, Electronic Structure, and Thermoelectric Properties of $\mathrm{MoS}_{2}$. J. Appl. Phys. 2013, 113, 013709. 